\title{
Coherent Optical Signatures of Electron Microbunching in Laser-Driven Plasma Accelerators
}

\author{
A. H. Lumpkin, ${ }^{1}$ M. LaBerge, ${ }^{2}$ D. W. Rule, ${ }^{3}$ R. Zgadzaj, ${ }^{2}$ A. Hannasch, ${ }^{2}$ O. Zarini $\odot,{ }^{4,5}$ B. Bowers $\odot,{ }^{2}$ \\ A. Irman, ${ }^{4}$ J. P. Couperus Cabadağ $\odot,{ }^{4}$ A. Debus, ${ }^{4}$ A. Köhler®, ${ }^{4,5}$ U. Schramm $\odot,{ }^{4,5}$ and M. C. Downer $\odot^{2, *}$ \\ ${ }^{1}$ Accelerator Division, Fermi National Accelerator Laboratory, Batavia, Illinois 60510, USA \\ ${ }^{2}$ Physics Department, University of Texas-Austin, Austin, Texas 78712, USA \\ ${ }^{3}$ Silver Spring, Maryland 20904, USA \\ ${ }^{4}$ Institute of Radiation Physics, Helmholtz-Zentrum Dresden-Rossendorf, 01328 Dresden, Germany \\ ${ }^{5}$ Technische Universität Dresden, 01062 Dresden, Germany
}

(Received 12 July 2019; revised 27 May 2020; accepted 9 June 2020; published 2 July 2020)

\begin{abstract}
We report observations of coherent optical transition radiation interferometry (COTRI) patterns generated by microbunched $\sim 200-\mathrm{MeV}$ electrons as they emerge from a laser-driven plasma accelerator. The divergence of the microbunched portion of electrons, deduced by comparison to a COTRI model, is $\sim 9 \times$ smaller than the $\sim 3 \mathrm{mrad}$ ensemble beam divergence, while the radius of the microbunched beam, obtained from COTR images on the same shot, is $<3 \mu \mathrm{m}$. The combined results show that the microbunched distribution has estimated transverse normalized emittance $\sim 0.4 \mathrm{~mm} \mathrm{mrad}$.
\end{abstract}

DOI: 10.1103/PhysRevLett.125.014801

Periodic longitudinal density modulation of relativistic electron beams ( $e$ beams) at optical wavelengths (microbunching) gives rise to coherent light emission in such forms as synchrotron radiation, including the free-electron laser (FEL) [1,2], and optical transition radiation (OTR) in its coherent form (COTR). The latter has been observed from FELs $[3,4]$ and laser-driven plasma accelerators (LPAs) [5-7]. The FEL mechanism depends fundamentally on growth of microbunching at the resonant wavelength and its harmonics [8]. In both FELs and LPAs, COTR can characterize microbunched portions of electrons [3-7]. Microbunching in an FEL oscillator was observed indirectly via buildup of FEL output power to saturation [2]. The first direct time-resolved observation of microbunching in an FEL oscillator [9] used an off-phase final rf accelerator stage to streak a beam modulated at $60-\mu \mathrm{m}$ wavelength, thereby mapping microbunch arrival time onto energy, measured in an electron spectrometer. With the advent of self-amplified spontaneous emission (SASE) FELs with a single pass through a long amplifier chain, FEL light and the $e$ beam became accessible after each undulator, enabling tracking of FEL power and microbunching. The first measurements of microbunching evolution at visible wavelengths $[3,4]$ used COTR interferometry (COTRI) [10] to track microbunched features

Published by the American Physical Society under the terms of the Creative Commons Attribution 4.0 International license. Further distribution of this work must maintain attribution to the author(s) and the published article's title, journal citation, and DOI. through exponential gain, saturation, and postsaturation [4]. An analytic model of COTRI fringe patterns showed growth of a microbunched transverse core in the exponential gain regime, and its subsequent reduction after saturation [4,11]. Subsequently, such experiments were extended to vacuum ultraviolet wavelengths, further benchmarking this model $[12,13]$. These experiments foreshadowed today's $x$ ray SASE FELs [2,14-18] by benchmarking the GENESIS simulation code [19] used in their prediction and development [20].

Here, we apply COTRI for the first time to LPAs [21], a class of compact accelerators that has been in scientific literature for 40 years [22], with compact LPA-driven FELs [23] now prominent among a growing list of their potential applications. Particle-in-cell simulations have predicted microbunching in LPAs [24,25], which might be used to seed an FEL. Indeed, faster start-up of a SASE FEL from a microbunched input beam than from noise has been reported [26]. Our measurements yield the first evaluation of beam emittance of the microbunched subset of LPA electrons with COTR techniques. Microbunching in LPAs is not accessible with betatron $\mathrm{X}$-ray spectroscopy [27], pepper-pot measurements [28], scintillator-based methods [29], or other LPA $e$-beam diagnostics [30]. Detailed understanding of microbunching is critical to developing LPAs and LPA-driven FELs. Past experiments used COTR to deduce the presence of subbunches located in adjacent LPA buckets [5] or of an intrabunch slice of subpercent energy spread [7]. Here, we use interference of COTR from two tandem foils downstream of the LPA to deduce the presence of visible-wavelength microbunching within the dominant quasimonoenergetic component of an electron 
bunch that was ionization-injected [31,32] into, and accelerated to $\sim 200 \mathrm{MeV}$ within, the leading plasma bubble of a strongly nonlinear LPA. Our results show that COTR gain relative to incoherent OTR rivals that obtained previously in a saturated SASE FEL. As a result, COTR is intense enough to distribute to multiple cameras with different frequency filters or imaging modalities on each shot, enabling thorough characterization. One group of cameras detects COTR imaged from the surface of the first foil [hereafter "near-field" (NF) images], which when analyzed using a coherent point-spread function determine beam size at the LPA exit. Other cameras record COTR in the focal plane of a collecting lens [hereafter "far-field" (FF) patterns], thereby measuring its angular distribution. Fringes in FF data, which are consistent with Wartski two-foil COTRI [10], fall outside of the $1 / \gamma$ emission cone angle of single-foil COTR. Here, $\gamma$ is the relativistic Lorentz factor of the electrons. High fringe visibility indicates microbunched electrons diverge significantly less than the $e$-beam ensemble. A COTRI model $[4,11]$ of FF data quantifies this divergence. Combined frequency-filtered beam-size and divergence data yield single-shot, transverse emittance estimates of the microbunched electrons.

Our experiments used pulses from the DRACO laser (central wavelength $800 \mathrm{~nm}$, energy up to $4 \mathrm{~J}$ on target, pulse length 27 fs FWHM) [32,33] at Helmholtz-Zentrum Dresden-Rossendorf. These were focused to $\sim 20 \mu \mathrm{m}$ (FWHM) at the center of a 3-mm-long He gas jet (with $3 \%$ nitrogen and $\sim 0.5$-mm-long entrance and exit ramps) to drive LPAs in a self-truncated ionization-injection regime [31] in plasma of density $n_{e} \sim 3 \times 10^{18} \mathrm{~cm}^{-3}$ [32]. Electron bunches with $(225 \pm 25)$-pC charge in a $200-\mathrm{MeV}(\gamma=400)$ quasimonoenergetic peak $\left(\sigma_{E}=0.042 \mathrm{rms}\right.$, or 10\% FWHM, ensemble energy spread) and $\sigma_{\theta}=3 \mathrm{mrad} \mathrm{rms}$ ensemble divergence, plus a weak polyenergetic background (see Fig. S.7b in Supplemental Material [34]) emerged from the LPA. A $75-\mu \mathrm{m}$-thick Al laser foil $700 \mu \mathrm{m}$ from the jet exit, tilted $\sim 3^{\circ}$ off normal [Fig. 1(a)], blocked the drive laser pulse. An aluminized Kapton ${ }^{\circledR}$ foil $1 \mathrm{~mm}$ downstream blocked $\mathbf{j} \times \mathbf{B}$ electrons of low energy and associated COTR from the back of the blocking foil [35] [Fig. 1(b)]. Indeed, direct laser excitation of the blocking foil with the gas jet turned off yielded no detectable OTR.

With the gas jet turned on, LPA electrons generated forward OTR/COTR from the aluminized back surface of the Kapton foil. Several dozen foil pairs were mounted on a $15-\mathrm{cm}$ diameter wheel, which rotated a fresh pair into position for each shot. A $200-\mu \mathrm{m}$-thick polished, nonaluminized $\mathrm{Si}$ wafer oriented at $45^{\circ}$ to the $e$ beam at distance $L=18.5 \mathrm{~mm}$ downstream of the Kapton foil redirected the foil's OTR/COTR to a 4-cm focal length microscope objective with collection angle $0.14 \mathrm{rad}$, which relayed it to one group of charge-coupled device (CCD) cameras (12-bit, 3.75- $\mu \mathrm{m}$ square pixels) via beam splitters [Fig. 1(a)] to record NF images [Fig. 1(c)]. Additionally,

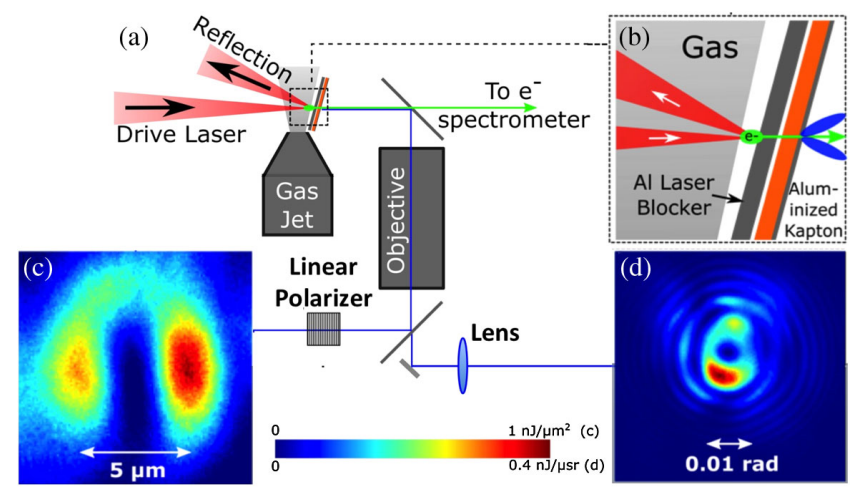

FIG. 1. (a) Schematic setup showing path of LPA drive laser pulse (red), gas jet, OTR foil, Si mirror, and OTR imagingdetection configuration. (b) Detail of gas jet exit, showing $e$ bunch, drive laser path, blocking foil, and aluminized Kapton OTR foil. (c) NF data at $600 \mathrm{~nm}$, imaged from Kapton foil to CCD through linear polarizer, showing two point-spread function lobes. (d) FF data at $633 \mathrm{~nm}$ for same shot, recorded with compound lens (microscope objective + single-element lens) and no polarization analyzer, showing interference fringes. Angular resolution: $0.35 \pm 0.05 \mathrm{mrad} / \mathrm{pixel}$, or $\sim 6 \mathrm{pixel} /$ fringe at eighth fringe. Color legend applies to (c) and (d).

the electron bunch generated reflected COTR from the Si surface [Fig. 1(a)]. COTR from these two surfaces, which the microscope objective and an additional $15-\mathrm{cm}$ focallength lens [see Fig. 1(a)] relayed to another CCD, formed interference fringes in FF images [Fig. 1(d)]. For results reported here, two CCDs recorded NF images through orthogonal linear polarization analyzers parallel $(x)$ and perpendicular $(y)$ to the drive laser polarization at $\lambda=600 \pm 5 \mathrm{~nm}$. These were indistinguishable from 633$\mathrm{nm}$ NF images. Generally, for a given shot, NF images differed significantly only when recorded at central wavelengths separated by $>\sim 50 \mathrm{~nm}$. Another CCD recorded unpolarized FF images at observation wavelength $\lambda=$ $633 \pm 5 \mathrm{~nm}$. Here, polarizers proved unnecessary because the ratio of $y$ - to $x$-polarized intensity along $\theta_{x}$ (or $x$ - to $y$-pol along $\theta_{y}$ ) was $\leq 0.025$ for all interference peaks (see Sec. 2e of Supplemental Material [34] for details).

When the laser focus in the gas jet was adjusted to positions that yielded polyenergetic electron distributions similar to the background in the present LPA output, but with no quasimonoenergetic peak, OTR signals weakened $\sim 10 \times$. Thus, the quasimonoenergetic peak is the dominant source of reported COTR signals in the ionization-injected regime. These signals were intense enough to necessitate neutral density filters to prevent camera saturation. When we operated the accelerator in different regimes-e.g., by removing the nitrogen dopant and relying on self-injection, or by introducing a shock front near the gas jet entrance to create a local density down ramp [36] — we observed strongly correlated variations in COTR signal strength normalized to accelerated charge. Section IV of Supplemental Material [34] summarizes our preliminary observations of these 
correlations. Since foil and laser parameters were unchanged, these variations suggested that the LPA process-not interaction of electrons with foils or reflected laser fields-created microbunching responsible for observed COTR. Beam scattering by a foil can reduce coherent emission from microbunching when the projected multiple scattering angle exceeds the OTR opening angle $1 / \gamma$ [37]. The latter is $2.3 \mathrm{mrad}$, while the Bethe-Ashkin formula [38] yields a lower value $(1.1 \mathrm{mrad})$ of the former for the $\mathrm{Al}$ foil, a higher value $(2.6 \mathrm{mrad})$ for the $45^{\circ} \mathrm{Si}$ mirror. To corroborate this conclusion, we measured space- and angleintegrated forward COTR spectra in a downstream IR-to-UV spectrometer [39]. With the Si wafer temporarily removed, we observed strong IR and visible light down to $\lambda \sim 300 \mathrm{~nm}$. When we reinserted the Si wafer and placed a new thin OTR foil downstream of it, however, only IR light remained strong. This showed that microbunching responsible for visible COTR survived transit through $\leq 75 \mu \mathrm{m} \mathrm{Al}$, but not $\geq 200 \mu \mathrm{m} / \cos 45^{\circ} \mathrm{Si}$ foils. We will present space-angleintegrated COTR spectra over a 5-octave spectral range in a planned forthcoming paper. Since FF images involve interference between COTR from two foils separated by $\sim 2 \mathrm{~cm}$, normalized emittance $\varepsilon_{n}$ and/or microbunching properties could change as a bunch with finite $\sigma_{E}$ propagates between them [40]. Explicit calculations, however, show that $\varepsilon_{n}$ grows by $<1 \%$ for our conditions (see Sec. 2a of Supplemental Material [34] for details). Moreover, we observed nearconstant single-foil integrated COTR intensity for $\mathrm{Al}$ foil positions over a $\sim 3 \mathrm{~cm}$ range from the LPA exit, consistent with findings of Lin et al. [7], suggesting that microbunching is stable within the COTRI apparatus.

Currents induced when a charged particle beam enters and exits a foil generate, respectively, backward and forward OTR in cones of half-angle $1 / \gamma$ around the specular reflection or beam direction [41-43]. Thus, the configuration in Fig. 1(a) generates OTR at $90^{\circ}$ to the beam direction, enabling minimally invasive OTR characterization. The number $W_{1}$ of OTR photons that a single electron generates per unit frequency $\omega$ per unit solid angle $\Omega$ in a single foil is

$$
\frac{d^{2} W_{1}}{d \omega d \Omega}=\frac{e^{2}}{\hbar c} \frac{1}{\pi^{2} \omega} \frac{\left(\theta_{x}^{2}+\theta_{y}^{2}\right)}{\left(\gamma^{-2}+\theta_{x}^{2}+\theta_{y}^{2}\right)^{2}},
$$

where $\hbar$ is Planck's constant $/ 2 \pi, e$ is electron charge, $c$ is the speed of light, and $\theta_{x}$ and $\theta_{y}$ are radiation angles [11]. The solid black curve in Fig. 2(a) shows the angular distribution of OTR at $\lambda=633 \pm 5 \mathrm{~nm}$ that a $200 \mathrm{MeV}$, $N$-electron bunch with divergence $\sigma_{x^{\prime}}=\sigma_{y^{\prime}} \equiv \sigma_{\theta}=$ $0.2 \mathrm{mrad}$ generates in one foil. There is one peak at $\theta \equiv \theta_{x}=\theta_{y}=1 / \gamma \sim 2.3 \mathrm{mrad}$, When $N_{B}$ of the $N$ particles are microbunched, a coherence function $\boldsymbol{J}(\boldsymbol{k})$ becomes involved, and for two foils an interference function $\boldsymbol{I}(\boldsymbol{k})$. The spectral angular distribution function then becomes

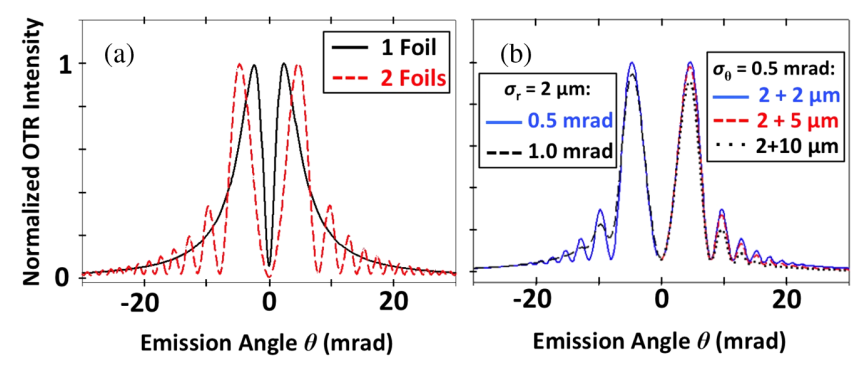

FIG. 2. (a) Analytical calculations of angular distributions of OTR at $\lambda=633 \pm 5 \mathrm{~nm}$ generated by $200 \mathrm{MeV}$ electron bunch with divergence $\sigma_{\theta}=0.2 \mathrm{mrad}$ : in one foil (solid black curve) and in a two-foil interferometer with $L=18.5 \mathrm{~mm}$ and beam radius $\sigma_{r}^{(1)}=\sigma_{r}^{(2)}=2 \mu \mathrm{m}$ at both foils 1 and 2 (dashed red). (b) Calculated dependence of COTRI fringe visibility on $\sigma_{\theta}$ and $\sigma_{r} . \theta<0$ : beam-divergence effect for $\sigma_{\theta}=0.5 \mathrm{mrad}$ (solid blue curve) and $1.0 \mathrm{mrad}$ (dashed black) for $\sigma_{r}^{(1)}=\sigma_{r}^{(2)}=2 \mu \mathrm{m}$. $\theta>0$ : effect of expanding $\sigma_{r}^{(2)}$ for fixed $\sigma_{r}^{(1)}=2 \mu \mathrm{m}$, $\sigma_{\theta}=0.5$-mrad, with curves labeled $\sigma_{r}^{(1)}+\sigma_{r}^{(2)} \mu \mathrm{m}$ in legend.

$\frac{d^{2} W}{d \omega d \Omega}=\left|r_{\|, \perp}\right|^{2} \frac{d^{2} W_{1}}{d \omega d \Omega}\left[N \boldsymbol{I}(\boldsymbol{k})+N_{B}\left(N_{B}-1\right) \boldsymbol{J}(\boldsymbol{k})\right]$,

where $\left|r_{\|, \perp}\right|^{2}$ is reflectance of the second ( $\mathrm{Si}$ ) foil for parallel, perpendicular polarization components, respectively. The reflectance reduces intensity of OTR from the first foil (via reflection from $\mathrm{Si}$ ) and OTR generated at the $\mathrm{Si}$ wafer [via Eq. (2)] equivalently. $\boldsymbol{I}(\boldsymbol{k})$ is [10]

$$
\boldsymbol{I}(\boldsymbol{k})=4 \sin ^{2}\left[\frac{k L}{4}\left(\gamma^{-2}+\theta_{x}^{2}+\theta_{y}^{2}\right)\right],
$$

where $k=|\boldsymbol{k}|=2 \pi / \lambda$, using a small-angle approximation. Peaks of $\boldsymbol{I}(\boldsymbol{k})$ occur at angles $\theta_{x}^{2}+\theta_{y}^{2}=(2 \lambda / L)\left(p-p_{0}\right)$, where $p=1 / 2,3 / 2, \ldots$, and $p_{0}=L /\left(2 \lambda \gamma^{2}\right)$. Adjacent peaks are separated by $\Delta\left(\theta_{x}^{2}+\theta_{y}^{2}\right)=2 \lambda / L$. Choosing $L=$ $18.5 \mathrm{~mm}$ provided good fringe contrast, while enabling near-field optics to focus on the first foil. The dashed red curve in Fig. 2(a) shows the two-foil OTR angular distribution for $L=18.5 \mathrm{~mm}$, with $\lambda, \gamma$, and $\sigma_{\theta}$ as for the black curve. Now there are multiple fringes. At this $\gamma$, tilt of the second foil introduces negligible intensity asymmetry, as is evident also from Eq. (1) of Ref. [42]. The coherence function can be defined as

$$
\boldsymbol{J}(\boldsymbol{k})=\left[H_{1}(\boldsymbol{k})-H_{2}(\boldsymbol{k})\right]^{2}+H_{1}(\boldsymbol{k}) H_{2}(\boldsymbol{k}) \boldsymbol{I}(\boldsymbol{k}),
$$

where $H_{j}(\boldsymbol{k})=\rho_{j}(\boldsymbol{k}) / Q=g_{j}\left(k_{x}\right) g_{j}\left(k_{y}\right) F\left(k_{z}\right)$ for an $e$ bunch of charge distribution $\rho_{j}(\boldsymbol{x})$ and total charge $Q$, with $j=1,2$. Here, we have introduced two microbunch form factors, $H_{1}$ and $H_{2}$, to account for the increase in bunch radius from the first to the second interferometer foil due to beam divergence. Each $H_{j}(\boldsymbol{k})$ is a product of Fourier transforms $g_{j}\left(k_{i}\right)=\exp \left(-\sigma_{i}^{2} k_{i}^{2} / 2\right)$ of transverse $(i=x, y)$ 
charge form factors (with $k_{i} \approx k \theta_{i}$ ), and of longitudinal form factor $F_{z}\left(k_{z}\right)=\exp \left(-\sigma_{z}^{2} k_{z}^{2} / 2\right)$, with $k_{z} \sim k$ and $\theta \ll 1$, assuming the Fourier transform $\rho_{j}(\boldsymbol{k})$ of $\rho_{j}(\boldsymbol{x})$ is separable. If $\boldsymbol{J}(\boldsymbol{k}) \ll 1$ or $N_{B} \rightarrow 0$, only the incoherent OTR term $(\sim N)$ remains in Eq. (2).

Figure 2(b) illustrates how transverse charge form factors determine COTR gain vs $\theta$, for azimuthally symmetric $\left(\sigma_{r}=\sigma_{x}=\sigma_{y}\right)$ beams of microbunched fraction $f_{B}=$ $N_{B} / N=0.01$. The left side $(\theta<0)$ shows effects of varying microbunched beam divergence $\left(\sigma_{\theta}=0.5,1.0 \mathrm{mrad}\right)$ alone, with $\sigma_{r}$ held fixed at $2 \mu \mathrm{m}$ at both foils; the right side $(\theta>0)$ shows the additional unavoidable effect of increased beam radius at foil $2\left(\sigma_{r}^{(2)}=2,5,10 \mu \mathrm{m}\right)$ for fixed $\sigma_{\theta}=0.5 \mathrm{mrad}$ and $\sigma_{r}^{(1)}=2 \mu \mathrm{m}$. As an example, for $\sigma_{r}^{(2)}=5-\mu \mathrm{m}, \sigma_{\theta}=0.5-\mathrm{mrad}, 8-9$ fringes are enhanced out to $\theta>20 \mathrm{mrad}$. In contrast, past COTRI studies of SASE FELs involved beam radii $25 \mu \mathrm{m}<\sigma_{x, y}<200 \mu \mathrm{m}$, and yielded at most 3-4 fringes out to only $\theta \sim 5 \mathrm{mrad}[4,11]$. LPA beams are often an order of magnitude smaller, so coherent enhancements can be proportionately larger and can extend to $\theta>30 \mathrm{mrad}$. Generally, optical detection bandwidth $\Delta \lambda$, electron energy bandwidth $\Delta \gamma, \sigma_{\theta}$, and $\sigma_{r}$, can all affect fringe visibility in a $\mathrm{FF}$ angular distribution pattern. Our chosen bandwidth filter $(\Delta \lambda=10 \mathrm{~nm})$, however, minimizes the bandwidth effect, while for our chosen $L$, the angles $\theta_{x, y}$ at which $I(k)$ peaks are, according to Eq. (3), nearly $\gamma$-independent for $300<\gamma<500$ (see Sec. 2d of Supplemental Material [34] for details). Thus, we can relate the observed fringe number directly to $\sigma_{\theta}$ and $\sigma_{r}$ of the contributing portion of the beam and observed COTR intensity directly to $f_{B}$. Consequently, observation of $\geq 3$ fringes implies submilliradian divergence and few-micrometer beam radius. Simultaneously, by calibrating integrated signal on the FF camera, we determine $J(\boldsymbol{k})$, and thus COTR gain $\left(\sim N_{B}^{2} / N\right.$ for $\left.N_{B} \gg 1\right)$ and $f_{B}$. We find $N=1.47 \times 10^{9}$ electrons in the quasimonoenergetic peak (i.e., $e N=$ $235 \mathrm{pC}$ ) and $N_{B}=1.8 \times 10^{7}$ (i.e., $e N_{B} \approx 3 \mathrm{pC}$ ), implying $f_{B} \approx 0.013$ and $N_{B}^{2} / N \approx 240000$. These results took into account single-electron, single-foil OTR source energy [44] and charge in the quasimonoenergetic peak determined from a calibrated LANEX® screen [45] in the spectrometer on the same shot (see Sec. I of Supplemental Material [34]). Thus, coherent enhancements dominate over incoherent OTR.

The FF image in Fig. 1(d) has eight to nine visible fringes. Azimuthally asymmetric fringes indicate a nonGaussian angular distribution of the COTR source. Here, to simplify analysis, we averaged this data azimuthally (Sec. 2b of Supplemental Material [34] describes the averaging procedure). Figure 3(a) compares the resulting fringe pattern (black curve) to analytical results. Since outer fringes [Fig. 3(a), inset] are more sensitive to $\sigma_{\theta}$ than inner fringes, whereas amplitudes of the latter are more sensitive to neglected non-Gaussian features of the beam angular distribution, we analyzed $\sigma_{\theta}$ by fitting COTRI model

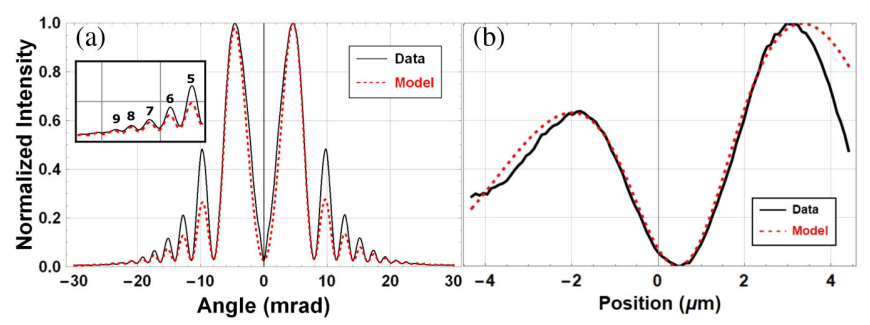

FIG. 3. (a) Azimuthally averaged COTRI fringes vs $\theta$ from Fig. 1(d) (solid black curve). See Supplemental Material [34] for description of averaging procedure. Inset: outermost fringes magnified. Dotted red curve: analytical COTRI model using $\sigma_{\theta}=0.33 \mathrm{mrad}$, and $\sigma_{x}=2.75 \mu \mathrm{m}$ and $6.6 \mu \mathrm{m}$ at foils 1 and 2 , respectively. (b) $x$ projection of NF image in Fig. 1(c) from same shot (solid black curve), with COTR model using $\sigma_{x}=2.75 \mu \mathrm{m}$ (dotted red curve).

calculations for various $\sigma_{\theta}$ to the fourth thru ninth fringes. Beam radii $\sigma_{r}^{(1)}$ and $\sigma_{r}^{(2)}$ also enter these calculations [see Fig. 2(b)]. We determined $\sigma_{r}^{(1)}$ independently by analyzing NF image Fig. 1(c) for the same shot. Figure 3(b) compares $y$-averaged, $x$-polarized NF data (black curve) to a COTR calculation for $\sigma_{x}^{(1)}=2.75 \mu \mathrm{m}$ (red dotted). Here, calculated curves take into account the optical collection angle via Eq. (26) of Ref. [46]. Left-right asymmetry of NF data probably results from distortions of $\rho(\boldsymbol{x})$ from a Gaussian shape, which we introduced into the model with an empirical skew parameter. Full analysis yields $\sigma_{x}^{(1)}=2.75_{-0.30}^{+0.45} \mu \mathrm{m}$. We generated statistics underlying the stated uncertainty by comparing single-shot data to an ensemble of single-parameter model curves (see Sec. III of Supplemental Material [34] for details). We then calculated $\sigma_{r}^{(2)}$ by adding $\sigma_{x}^{(1)}$ and $\sigma_{\theta} L$ in quadrature. Finally, we fit the black curve in Fig. 3(a) to an ensemble of model curves, with $\sigma_{\theta}$ as the sole free parameter, and minimized $\chi^{2}$. The dotted red curve in Fig. 3(a) shows the best-fit COTRI curve. Full analysis (see Sec. 2f of Supplemental Material [34]), yields $\sigma_{\theta}=0.33_{-0.05}^{+0.12} \mathrm{mrad}$, $9 \times$ smaller than the ensemble divergence [32]. Only submilliradian $\sigma_{\theta}$ can explain 8 to 9 visible fringes. When combined with $\sigma_{x}^{(1)}$, we estimate normalized emittance $\varepsilon_{n x}=$ $0.36_{-0.09}^{+0.19} \mathrm{~mm} \mathrm{mrad}$ (rms) for the microbunched beam portion at foil 1. $y$-polarized NF data yielded similar values. Significant position-momentum correlation, if present, would reduce this emittance value [47].

To illustrate more complex beam structure, and wider COTRI diagnostic possibilities, Fig. 4(a) shows FF data for a different shot. Here, a dark node runs vertically through the interference pattern, a feature not seen in Fig. 1(d), nor in most shots. The corresponding NF image [Fig. 4(b)] shows two pairs of point-spread-function lobes separated by $\sim 6 \mu \mathrm{m}$ along $x$, instead of one pair as in Fig. 1(c), suggesting that two beamlets emerged side by side from the LPA, as seen in some simulations of bubble-regime LPAs [48]. Figure 4(c) shows a reconstruction of the main 


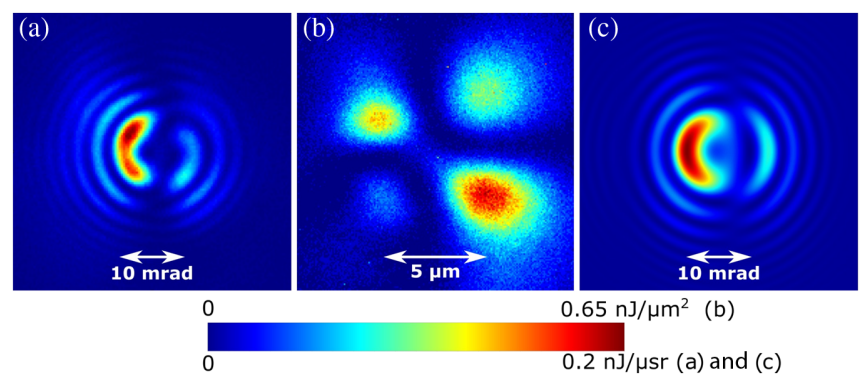

FIG. 4. (a) COTR angular distribution pattern for shot with vertical dark band due to interference of two beamlets. (b) $y$ polarized NF image on same shot, showing two double-lobed patterns. (c) COTRI image reconstructed from two-beamlet model. Color legend applies to all panels.

qualitative features of Fig. 4(a) by modeling two beamlets, each with $\sigma_{\theta}=0.6 \mathrm{mrad}$, with phase and angular trajectories differing by $0.75 \pi$ and $2 \mathrm{mrad}$, respectively. This example illustrates that FF COTRI patterns contain signatures of the phase and trajectory of multiple microbunched beamlets, when present. Such bunch pairs, if controllable, could open new LPA applications-e.g., pump-probe experiments or two-color FELs. Single-shot diagnosis demonstrated here could help develop control methods for generating bunch pairs reliably.

In summary, we identified microbunched $e$ beamlets from LPAs, which generate visible COTR $>10^{5}$ times stronger than incoherent OTR. We discovered and modeled COTRI fringes, and applied COTRI, along with COTR imaging and calibrated COTR energy measurements, for the first time to LPAs. This coordinated triad of COTR measurements revealed divergences and emittances of microbunched beamlets that are less than those of the electron ensemble, which motivates SASE FEL startup experiments. Although single-wavelength COTR measurements alone do not distinguish periodic longitudinal density modulation from sharp density spikes, timedomain reconstructions from space-angle-integrated COTR spectra [39] and simulations of ionization-injected LPAs [24] indicate that microbunched beamlets studied here were longitudinally modulated. Future directions include understanding correlations among COTR intensity, LPA operating regime, and microbunching mechanisms, and developing strategies for diagnosing, tuning, and optimizing microbunching over a wide range of wavelengths.

A. H. L. acknowledges discussions with K.-J. Kim (ANL) on microbunching in FELs and R. ThurmanKeup (FNAL) on COTRI, and support from Fermi Research Alliance under Contract No. DE-AC0207CH11359 with the U.S. Department of Energy, Office of Science, Office of High Energy Physics. U. Texas authors acknowledge support from DOE Grant No. DE-SC0011617, and M.C.D. from the Alexander von Humboldt Foundation with sponsorship from R. Sauerbrey. Helmholtz-Zentrum Dresden-Rossendorf authors acknowledge support from the Helmholtz Association under program Matter and Technology, topic Accelerator R\&D.

*downer@physics.utexas.edu

[1] J. M. J. Madey, Stimulated emission of Bremsstrahlung in a periodic magnetic field, J. Appl. Phys. 42, 1906 (1971).

[2] K.-J. Kim, Z. Huang, and R. Lindberg, Synchrotron Radiation and Free-Electron Lasers: Principles of Coherent X-Ray Generation (Cambridge University Press, Cambridge, England, 2017).

[3] A. H. Lumpkin, R. Dejus, W. J. Berg, M. Borland, Y. C. Chae, E. Moog, N. S. Sereno, and B.X. Yang, First Observation of $z$-Dependent Electron-Beam Microbunching Using Coherent Transition Radiation, Phys. Rev. Lett. 86, 79 (2001).

[4] A. H. Lumpkin et al., Evidence for Microbunching "Sidebands" in a Saturated Free-Electron Laser Using Coherent Optical Transition Radiation, Phys. Rev. Lett. 88, 234801 (2002).

[5] Y. Glinec, J. Faure, A. Norlin, A. Pukhov, and V. Malka, Observation of Fine Structures in Laser-Driven Electron Beams Using Coherent Transition Radiation, Phys. Rev. Lett. 98, 194801 (2007).

[6] O. Lundh et al., Few femtosecond, few kiloampere electron bunch produced by a laser-plasma accelerator, Nat. Phys. 7, 219 (2011).

[7] C. Lin et al., Long-Range Persistence of Femtosecond Modulations on Laser-Plasma-Accelerated Electron Beams, Phys. Rev. Lett. 108, 094801 (2012).

[8] K.-J. Kim, An analysis of self-amplified spontaneous emission, Nucl. Instrum. Methods Phys. Res., Sect. A 250, 396 (1986).

[9] K. N. Ricci and T. I. Smith, Longitudinal electron beam and free electron laser microbunch measurements using offphase rf acceleration, Phys. Rev. ST Accel. Beams 3, 032801 (2000).

[10] L. Wartski, S. Roland, J. Lasalle, M. Bolore, and G. Fillippi, Interference phenomenon in optical transition radiation and its application to particle beam diagnostics and multiplescattering measurements, J. Appl. Phys. 46, 3644 (1975).

[11] D. W. Rule and A. H. Lumpkin, Analysis of coherent optical transition radiation interference patterns produced by SASE-induced microbunches, in Proceedings of the 2001 Particle Accelerator Conference (IEEE, Piscataway, NJ, 2001), Vol. 2, pp. 1288-1290.

[12] A. H. Lumpkin, R. J. Dejus, and D. W. Rule, First direct comparisons of a COTRI analytical model to data from a SASE FEL at 540-nm, 265-nm, and 157-nm, in Proceedings, 26th International Conference, Free Electron Laser 2004 (Comitato Conferenze Elettra, Treiste, Italy, 2004), pp. 519-522.

[13] A. H. Lumpkin, Y.-C. Chae, R. J. Dejus, M. Erdmann, J. W. Lewellen, and Y. Li, Use of VUV imaging to evaluate COTR and beam-steering effects in a SASE FEL at $130 \mathrm{~nm}$, in Proceedings, 26th International Conference, Free Electron 
Laser 2004 (Comitato Conferenze Elettra, Treiste, Italy, 2004), pp. 523-526.

[14] J. Arthur et al., Linac Coherent Light Source (LCLS) conceptual design report, SLAC Report SLAC-R-593, 2002.

[15] JASRI/Spring-8, and Harima RIKEN, SPring-8 compact SASE source conceptual design report, edited by T. Tanake and T. Shintake (RIKEN Harima Institute, Hyogo, 2005).

[16] H.-S. Kang, K.-W. Kim, and I.-S. Ko, Current status of the PAL-XFEL project, in Procceedings, International Particle Accelerator Conference 2014 (JACoW Publishing, Dresden, Germany, 2014), pp. 2897-2899.

[17] R. Brinkman, B. Faatz, K. Flöttmann, J. Rossbach, J. R. Schneider, H. Schult-Schrepping, D. Trines, T. Tschentscher, and H. Weise, TESLA XFEL: First stage of the X-ray laser laboratory, DESY, Report TESLA FEL2002-09, 2002.

[18] Paul Scherrer Institute (PSI), Swiss FEL conceptual design report, edited by R. Ganter (Paul Scherrer Institute, Villigen, 2010).

[19] S. Reiche, GENESIS 1.3: a fully 3D time-dependent FEL simulation code, Nucl. Instrum. Methods Phys. Res., Sect. A 429, 243 (1999).

[20] S. V. Milton et al., Exponential gain and saturation of a selfamplified spontaneous emission free-electron laser, Science 292, 2037 (2001).

[21] S. M. Hooker, Developments in laser-driven plasma accelerators, Nat. Photonics 7, 775 (2013).

[22] T. Tajima and J. M. Dawson, Laser Electron Accelerator, Phys. Rev. Lett. 43, 267 (1979).

[23] F. Gruener et al., Design considerations for table-top, laserbased VUV and X-ray free electron lasers, Appl. Phys. B 86, 431 (2007); K. Nakajima, Towards a table-top free-electron laser, Nat. Phys. 4, 92 (2008); T. André et al., Control of laser plasma accelerated electrons for light sources, Nat. Commun. 9, 1334 (2018).

[24] X. L. Xu et al., Nanoscale Electron Bunching in LaserTriggered Ionization Injection in Plasma Accelerators Phys. Rev. Lett. 117, 034801 (2016).

[25] K. Németh, B. Shen, Y. Li, H. Shang, R. Crowell, K. C. Harkay, and J. R. Cary, Laser-Driven Coherent Betatron Oscillation in a Laser-Wakefield Cavity, Phys. Rev. Lett. 100, 095002 (2008).

[26] A. H. Lumpkin, R. J. Dejus, and N. S. Sereno, Coherent optical transition radiation and self-amplified spontaneous emission generated by chicane-compressed electron beams, Phys. Rev. ST Accel. Beams 12, 040704 (2009).

[27] G. R. Plateau et al., Low-Emittance Electron Bunches from a Laser-Plasma Accelerator Measured using Single-Shot X-Ray Spectroscopy, Phys. Rev. Lett. 109, 064802 (2012).

[28] E. Brunetti et al., Low Emittance, High Brilliance Relativistic Electron Beams from a Laser-Plasma Accelerator, Phys. Rev. Lett. 105, 215007 (2010); A. Cianchi et al., Challenges in plasma and laser wakefield accelerated beams diagnostic, Nucl. Instrum. Methods Phys. Res., Sect. A 720, 153 (2013).

[29] S. K. Barber et al., Measured Emittance Dependence on the Injection Method in Laser Plasma Accelerators, Phys. Rev. Lett. 119, 104801 (2017).

[30] M. C. Downer, R. Zgadzaj, A. Debus, U. Schramm, and M. C. Kaluza, Diagnostics for plasma-based electron accelerators, Rev. Mod. Phys. 90, 035002 (2018).
[31] M. Mirzaie et al., Demonstration of self-truncated ionization injection for GeV electron beams, Sci. Rep. 5, 14659 (2015).

[32] J. Couperus et al., Demonstration of a beam loaded nanocoulomb-class laser wakefield accelerator, Nat. Commun. 8, 487 (2017); A. Irman, J. P. Couperus, A. Debus, A. Köhler, J. M. Krämer, R. Pausch, O. Zarini, and U. Schramm, Improved performance of laser wakefield acceleration by tailored self-truncated ionization injection, Plasma Phys. Controlled Fusion 60, 044015 (2018).

[33] U. Schramm et al., First results with the Novel Peta-Watt laser acceleration facility in Dresden, in Proceedings, International Particle Accelerator Conference 2017 (IOP Publishing, Copenhagen, Denmark, 2017); J. Phys. Conf. Ser. 874, 012028 (2017).

[34] See Supplemental Material at http://link.aps.org/ supplemental/10.1103/PhysRevLett.125.014801 for details of COTR gain calibration, data analysis and correlation with LPA regime.

[35] H. Popescu, D. D. Baton, and F. Amiranoff, Subfemtosecond, coherent, relativistic, and ballistic electron bunches generated at $\omega_{0}$ and $2 \omega_{0}$ in high intensity laser-matter interaction, Phys. Plasmas 12, 063106 (2005).

[36] K. Schmid, A. Buck, C. M. S. Sears, J. M. Mikhailova, R. Tautz, D. Herrmann, M. Geissler, F. Krausz, and L. Veisz, Density-transition based electron injector for laser driven wakefield accelerators, Phys. Rev. ST Accel. Beams 13, 091301 (2010).

[37] A. Murokh, E. Hemsing, and J. Rosenzweig, Multiple Scattering-Induced Mitigation of COTR Emission from Microbunched Electron Beams, in Proceedings, Particle Accelerator Conference 2009 (JACoW Publishing, Vancouver, BC, Canada, 2009), p. 3991.

[38] E. Segrè et al., Experimental Nuclear Physics (John Wiley and Sons, NY, 1953), Vol. I, p. 285.

[39] O. Zarini et al., Advanced methods for temporal reconstruction of modulated electron bunches, in Proceedings, Advanced Accelerator Concepts Workshop 2018 (IEEE, Breckenridge, CO, USA, 2018).

[40] M. Migliorati, A. Bacci, C. Benedetti, E. Chiadroni, M. Ferrario, A. Mostacci, L. Palumbo, A. R. Rossi, L. Serafini, and P. Antici, Intrinsic normalized emittance growth in laserdriven electron accelerators, Phys. Rev. ST Accel. Beams 16, 011302 (2013).

[41] V. L. Ginzburg and I. M. Frank, Radiation of a uniformly moving electron due to its transition from one medium into another, Sov. Phys. JETP 16, 15 (1946).

[42] D. W. Rule, Transition radiation diagnostics for intense charged particle beams, Nucl. Instrum. Methods Phys. Res., Sect. B 24-25, 901 (1987).

[43] A. H. Lumpkin, in Proceedings, Accelerator Instrumentation Workshop 1990 (American Institute of Physics, College Park, 1991); Advanced, time-resolved imaging techniques for electron-beam characterizations, AIP Conf. Proc. 229, 151 (1991).

[44] C. B. Schroeder, E. Esarey, J. van Tilborg, and W. P. Leemans, Theory of coherent transition radiation generated at a plasmavacuum interface, Phys. Rev. E 69, 016501 (2004).

[45] T. Kurz et al., Calibration and cross-laboratory implementation of scintillating screens for electron bunch charge determination, Rev. Sci. Instrum. 89, 093303 (2018). 
[46] M. Castellano and V. A. Verzilov, Spatial resolution in optical transition radiation beam diagnostics, Phys. Rev. ST Accel. Beams 1, 062801 (1998).

[47] A. Curcio et al., Trace-space reconstruction of low-emittance electron beams through betatron radiation in laser-plasma accelerators, Phys. Rev. Accel. Beams 20, 012801 (2017).
[48] A. H. Lumpkin, R. Crowell, Y. Li, and K. Nemeth, A compact electron spectrometer for an LWFA, in Proceedings, 29th International Conference, Free Electron Laser 2007 (Novosibirsk, Russia, 2007), p. 294, www.JACoW .org. 